'RN Said, ${ }^{1} \mathrm{Y}$ Mansi, ${ }^{2} \mathrm{AM}$ Fattouh, ${ }^{2} \mathrm{RI}$ Esmaeel, ${ }^{3} \mathrm{H}$ Mahmoud. ${ }^{1}$ Pediatrics \& Neonatology, Faculty of Medicine Cairo University; ${ }^{2}$ Pediatrics \& Cardiology; ${ }^{3}$ Clinical Pathology, Faculty of Medicine - Cairo University, Cairo, Egypt

Background and Aim The role of $\mathrm{N}$ terminal pro- B type natriuretic peptide (NT-pro-BNP) to differentiate cardiac and respiratory causes of dyspnea in adults has been previously investigated. This study is conducted in order to evaluate the diagnostic value of this peptide in differentiating between cardiac and respiratory causes of neonatal respiratory distress.

Methods A prospective case-control study was conducted on 30 neonates $>34$ weeks gestational age, presenting with signs of respiratory distress who were evaluated clinically and underwent NTpro-BNP assay on the $4^{\text {th }}$ and on the 10th days of life if respiratory symptoms continued. Echocardiography was performed for all cases and accordingly classified into cardiac and respiratory problems ( $C P \& R P)$ groups based on the presence of significant cardiac defects. The control group included 17 healthy neonates.

Results Each of the CP and RP groups included 15 infants. The mean value of NT-pro-BNP was significantly higher in the CP group than the RP group on the $4^{\text {th }}$ day of life. The best calculated cut-off point was $196.4 \mathrm{fmol} / \mathrm{L}$ (95\% CI $61.7-95.2 \%$, sensitivity of $73.3 \%$ and specificity of $64.3 \%$ ). A level of $127 \mathrm{fmol} / \mathrm{L}$ could be used to rule out cardiac disease (sensitivity of $100 \%$ and specificity of $37 \%$ ). A level of $480 \mathrm{fmol} / \mathrm{L}$ can be used to rule in cardiac disease (sensitivity of $46 \%$ and specificity of $100 \%$ ). In between these 2 levels, there is a grey zone with 5 cases (33.3\%).

Conclusions NT-pro-BNP levels can be a useful biomarker to identify neonates with cardiac problems.

\section{A NEUROBEHAVIORAL INTERVENTION AND ASSESSMENT PROGRAM IN VERY LOW BIRTH WEIGHT INFANTS: OUTCOME AT 5 YEARS OF CORRECTED AGE}

doi:10.1136/archdischild-2012-302724.0324

'JWP van Hus, 'M JeukensVisser, ' $\mathrm{K}$ Koldewijn, ${ }^{2} \mathrm{CJA}$ Geldof, ${ }^{3} \mathrm{JH}$ Kok, ${ }^{3} \mathrm{AG}$ van WassenaerLeemhuis, ' $\mathrm{F}$ Nollet. 'Rehabilitation, Academic Medical Center; ${ }^{2} \mathrm{Clinical}$ Neuropsychology, VU University Medical Center; ${ }^{3}$ Neonatology, Emma's Children Hospital, Academic Medical Center, Amsterdam, The Netherlands

Background We carried out a RCT to evaluate the effect of the Infant Behavioral Assessment and Intervention Program (IBAIP) in 176 VLBW infants. This post discharge intervention program was given until 6 months CA. Positive intervention effects were found on mental outcome at 6 and on motor outcome at 6,24 , and 44 months. Aim To evaluate the effect of the IBAIP in VLBW infants on cognitive, neuromotor, and behavioral development at 5.5 years CA.

Methods Development was assessed using the Wechsler Preschool and Primary Scale of Intelligence (WPPSI-III-NL), the Movement Assessment Battery for Children (MABC-2), the Developmental Test of Visual Motor Integration (VMI), a neurological examination, and the Strength and Difficulties questionnaire (SDQ).

Results Sixty-nine VLBW children in the intervention and 67 VLBW children in the control group participated at 5.5 years CA (response rate $77.3 \%$ ). Some important social and perinatal risk factors were at the disadvantage of the intervention group. Verbal and performance IQ-scores < 85 occurred significantly less often in the intervention group ( $17.9 \%$ versus $33.3 \%, \mathrm{p}=0.041$, and $7.5 \%$ versus $21.2 \%, p=0.023$, respectively). After adjustment, only the odds ratio for performance IQ was significant: $0.24,95 \%$ CI: 0.06-0.95. Significant intervention effects on mean scores were found on WPPSI-IIINL subtasks block design and vocabulary. After adjustment, mean scores were significantly better in the intervention group on these WPPSI-III-NL subtasks, MABC-2 component aiming and catching and the VMI.

Conclusions The IBAIP leads to improvement in intelligence, ball skills and visual-motor integration at 5.5 years CA.

\section{ATTENTION PROBLEMS IN VERY LOW BIRTH WEIGHT PRESCHOOLERS}

doi:10.1136/archdischild-2012-302724.0325

${ }^{1} \mathrm{G}$ Verkerk, ${ }^{2} \mathrm{~B}$ Houtzager, ${ }^{\mathrm{M}} \mathrm{M}$ JeukensVisser, ${ }^{3} \mathrm{~A}$ van WassenaerLeemhuis, ' $\mathrm{K}$ Koldewijn, 'F Nollet, ${ }^{3} \mathrm{~J}$ Kok. 'Rehabilitation; ${ }^{2} E m m a$ Children's Hospital; ${ }^{3}$ Neonatology, Academic Medical Center, University of Amsterdam, Amsterdam, The Netherlands

Aims To compare very low birth weight (VLBW) children's performance on attention measurements at preschool age to term-born peers, and to assess associated risk factors.

Methods Attention was assessed in 151 VLBW preschoolers and 41 term-born peers at 44 months of corrected age, using the Visual Attention task of the Developmental Neuropsychological Assessment, and the Gift Delay task. Parents completed the Attention Problems domain of the Child Behavior Checklist (CBCL-AP), the Inhibit domain of the Behavior Rating Inventory of Executive Function (preschool version), and the Inattention/Distractibility scale of the Sensory Profile. The investigator completed the Attention domain of the Miller Assessment for Preschoolers' Behavior During Testing (BDT-AD). Potential perinatal and socio-demographic risk factors for attention problems were analyzed using logistic regression analysis.

Results Compared with term-born peers, VLBW children scored worse on five out of six attention measurements and had significantly more abnormal scores on the CBCL-AP and BDT-AD. Analyses of BDT-AD indicate that VLBW children mostly scored abnormally on the items regarding attention maintenance. VLBW children were at higher risk for attention problems according to a composite score of significant attention problems (OR 4.6, 95\% CI: 1.7-12.4). Risk factors for attention problems were having a mother born abroad (OR 3.5, CI: 1.7-7.2) and bronchopulmonary dysplasia (BPD) (OR 2.5, CI: 1.0-6.0).

Conclusions At the time of school entry, VLBW children have more difficulty maintaining attention than their term-born peers. Both social and biological risk factors were predictive of attention problems. Using the CBCL-AP and BDT-AD may lead to timely intervention

\section{VISUAL SENSORY AND PERCEPTIVE FUNCTIONS IN VERY LOW BIRTHWEIGHT (VLBW) PRESCHOOLERS}

doi:10.1136/archdischild-2012-302724.0326

${ }^{1,2} \mathrm{CJA}$ Geldof, ${ }^{3} \mathrm{AG}$ van Wassenaer, ${ }^{3} \mathrm{JH}$ Kok, ${ }^{1} \mathrm{~J}$ Oosterlaan. 'Department of Clinical Neuropsychology, VU University Amsterdam; ${ }^{2}$ Rehabilitation and Advice, Royal Dutch Visio; 'Department of Neonatology, Emma's Children Hospital, Academic Medical Center, Amsterdam, The Netherlands

Introduction Recent meta-analytic findings show impaired visual perceptive performance for VLBW children. Little is known about relationships between visual sensory and visual perceptive processes in VLBW children.

Methods VLBW children $(n=121)$ and age matched controls $(n=50)$ were assessed using tests for oculomotor functioning (eye position, motility, convergence, nystagmus and torticollis), visual sensory functions (visual acuity, visual field, contrast sensitivity, color perception and stereovision) and visual perceptive abilities (form and motion coherence, Position in Space, Figure-Ground, Visual Closure Form Constancy and face perception).

Results Compared to term born controls, VLBW children showed more disorders of eye position $(p=0.01)$ and convergence $(p=0.03)$ For visual sensory functions, VLBW children had lower single symbol $(p<0.001)$, but not different line symbol $(p=0.06)$ visual acuity and displayed reduced or absent stereovision more often $(p=0.04)$. Visual perceptive tasks showed reduced performance on both form and motion coherence tasks $(p=0.01)$ and on the subtests Position in Space $(p=0.001)$, Figure-Ground $(p=0.002)$, and Visual Closure 